\title{
Chronic administration of green tea extract to TRAMP mice induces the collapse of Golgi apparatus in prostate secretory cells and results in alterations of protein post-translational processing
}

\author{
PIERPAOLA DAVALLI $^{1^{*}}$, FEDERICA RIZZI ${ }^{2 *}$, GAETANO FELICE CALDARA $^{3}$, SERENA DAVOLI $^{2}$, \\ ARNALDO CORTI $^{1}$, ALESSANDRO SILVA ${ }^{2}$, SERENELLA ASTANCOLLE ${ }^{1}$, MARCO VITALE $^{3}$, \\ SAVERIO BETTUZZI ${ }^{2}$, MARIALUISA ARCARI ${ }^{3}$ and GIACOMO AZZALI ${ }^{3}$
}

\author{
${ }^{1}$ Department of Biomedical Sciences, University of Modena and Reggio Emilia, I-41100 Modena; ${ }^{2}$ Department of \\ Experimental Medicine, University of Parma, I-43125 Parma and INBB, Istituto Nazionale Biostrutture e Biosistemi, I-00136 \\ Rome; ${ }^{3}$ Department of Human Anatomy, Pharmacology and Forensic Medicine, University of Parma, I-43125 Parma, Italy
}

Received May 20, 2011; Accepted June 22, 2011

DOI: 10.3892/ijo.2011.1136

\begin{abstract}
Considering its long latency, prostate cancer (PCa) represents an ideal target for chemoprevention strategies. Green tea extract (GTE) has been proved to be one of the most promising natural substances capable of inhibiting PCa progression in animal models (transgenic adenocarcinoma of mouse prostate), as well as in humans. However, the cellular targets of the GTE action are mostly unknown. The main objective of this work was to investigate whether the endoplasmic reticulum (ER) and the Golgi apparatus (GA), known to be actively involved in sensing stress stimuli and initiating and propagating cell death signalling, may represent the subcellular targets of GTE action. To this end, 42 TRAMP mice were divided into four experimental groups: groups II and IV, received GTE in tap water $(0.3 \mathrm{~g} / 100 \mathrm{ml}$ solution) starting at 8 weeks of age and up to the time of sacrifice. Groups I and III were respective agematched water-fed controls. The animals were sacrificed after 4 weeks (groups I and II) or 40 weeks of treatment (groups II and IV). We also treated TRAMP-C2 cells with GTE (20 $\mu \mathrm{g} / \mathrm{ml}$ for 7 days) to check the expression profile of clusterin (CLU), a protein involved in prostate tumourigenesis, extensively processed through ER-GA before being secreted through the plasma membrane. In vivo we found that chronic administration of GTE in TRAMP mice results in collapse of ER and GA in prostate epithelial cells. Consistently, in vitro we found that the mature, fully processed form of CLU, sCLU, is strongly reduced by GTE treatment in TRAMP-C 2 cells. Taking into
\end{abstract}

Correspondence to: Professor Saverio Bettuzzi, Department of Experimental Medicine, Faculty of Medicine, University of Parma, Via Volturno 39/a, 43125 Parma, Italy

E-mail: saverio.bettuzzi@unipr.it

${ }^{*}$ Contributed equally

Key words: green tea extract, chemoprevention, prostate cancer, clusterin, ultrastructure, transgenic adenocarcinoma of mouse prostate account the sCLU biogenesis dependence on the ER-GA integrity and the proposed anti-apoptotic role of sCLU, the possibility for GTE to counteract PCa progression by interfering with sCLU biogenesis is suggested.

\section{Introduction}

Genetically engineered animal models have emerged as fundamental tools to study many pathological conditions, including cancer, and to find new therapeutic strategies. The transgenic adenocarcinoma of mouse prostate (TRAMP) is an experimental model for prostate cancer ( $\mathrm{PCa})$, which closely mimics the pathogenesis of the human disease. TRAMP mice express SV40 T/t antigen under the control of the prostate-specific minimal rat probasin promoter (1). In fact, it represents a genetically defined paradigm in which to investigate the stochastic molecular progression of prostate cancer from prostatic intraepithelial neoplasia (PIN), through well differentiated localized disease, to invasive and metastatic androgen-independent cancer $(1,2)$. Many detailed anatomical and histological studies of TRAMP mouse prostate have been reported since this model was created by Greenberg in 1995 (1-7) showing quantitative analysis of the incidence and distribution of pathological lesions as a function of aging.

Numerous studies, by us and others, indicate that $\mathrm{PCa}$ development in TRAMP mice as well as in humans, is inhibited or delayed by green tea extracts (GTE) administration (8-12); to our knowledge, however, a systematic description of the morphological changes of the prostate epithelium associated with GTE consumption is still missing.

Here we used the transmission electron microscopy (TEM) analysis to explore possible structural changes that might occur at the level of the intimate morphology of the secretory epithelial components in the prostatic acinar adenomeres of TRAMP mice. The study was conducted during the development of PCa, either as a function of aging and following the chemopreventive GTE treatment.

Very recent convincing evidence indicates that the secretory pathway, i.e. the endoplasmic reticulum (ER) and the 
Golgi apparatus (GA), can be actively involved in sensing stress stimuli and even initiating and propagating cell death signaling (13), which may represent a new interesting drug target for possible therapeutic interventions. Based on this consideration, we focused our microscopic observation mainly on the secretory pathway, assuming that GTE might affect the activity of the ER-GA, thus deregulating protein trafficking through the cytoplasm.

In order to explore this hypothesis with a multidisciplinary approach, we paralleled our in vivo experiments with in vitro ones using TRAMP-C2 cells a syngeneic prostate cancer cell line established from a heterogeneous tumor of the TRAMP model (14). In these cells we studied the effect of chronic GTE administration on the maturation of proteins processed through the secretory pathway. More precisely we characterized, by Western blot analysis, the pattern of expression of clusterin (CLU), a paradigmatic secreted protein, involved in PCa development $(15,16)$, extensively processed in the ER-GA after translation of the primary transcript $(17,18)$. CLU processing, includes heavy glycosylation of a precursor protein (pCLU) and cleavage to an $\alpha-\beta$ dimer before being secreted through the plasma membrane (sCLU) (17). It is well known that CLU post-translational processing is deeply affected by many conditions involving ER-GA stress $(19,20)$. Although CLU-specific role in tumourigenesis is still a matter of debate $(15,16,21)$, there is common consent on the cyto-protective, pro-survival role of sCLU (22). Thus, the present paper represents, to our knowledge, the first morphological ultrastructural study of the TRAMP mouse prostatic secretory cells as a function of disease progression and following GTE administration.

The experimental data reported here suggest that in TRAMP mouse prostatic secretory cells a mechanism may exist through which GTE affects the condensing and secretory activities of the ER-GA. This in turn would affect CLU processing causing a reduction of sCLU production, thus blocking the cyto-protective action of the protein on prostate cells.

\section{Materials and methods}

Animals. TRAMP mice, heterozygous for the PB-Tag transgene were obtained as (TRAMP x C57BL/6)F1 or F2 offspring and maintained in a C57BL/6 background. The animals were housed according with protocols approved by the institutional laboratory animal care and use committee of Italy. Tail tips from all male offspring were genotyped by polymerase chain reaction to identify mice carrying the transgene as previously described (1).

For the present research we used 42 mice assigned to 4 experimental groups identified with roman numerals (Table I). The animals had access to laboratory chow and water ad libitum. Animals belonging to the groups II and IV, received GTE in tap water $(0.3 \mathrm{~g} / 100 \mathrm{ml}$ solution) as previously made (10), starting at the age of 8 weeks until the sacrifice. Animals belonging to the groups I and III represent their respective age-matched waterfed controls. The $0.3 \%$ GTE solution was freshly prepared and replaced every Monday, Wednesday and Friday. GTE treatment did not affect either the animal weight, or the SV40 tag expression. At established time (12 and 48 weeks of age) the animals were sacrificed, according to the rules of the Ethics Committee, under ether anaesthesia.
Table I. Description of the experimental groups.

\begin{tabular}{lccc}
\hline $\begin{array}{l}\text { Group } \\
\text { name }\end{array}$ & Animals no. & $\begin{array}{c}\text { GTE treatment } \\
\text { (time) }\end{array}$ & $\begin{array}{c}\text { Age of sacrifice } \\
\text { (weeks) }\end{array}$ \\
\hline Group I & 9 & No & 12 \\
Group II & 9 & Yes (4 weeks) & 12 \\
Group III & 12 & No & 48 \\
Group IV & 12 & Yes (40 weeks) & 48 \\
\hline
\end{tabular}

Green tea extract. TRAMP mice and TRAMP-C2 prostate cancer cells were treated with Polyphenon- $\mathrm{E}^{\circledR}$, a standardized green tea polyphenols extract produced by Mitsui Norin, Shizuoka, Japan and kindly provided by Polyphenon Pharma, New York, USA. The mixture, hereafter referred as GTE, contains (-)-epigallocatechin-3-gallate (54.8\%), (-)-epigallocatechin $(9.2 \%)$, (-)-epicatechin $(10.8 \%),(-)$-epicatechin-3-gallate $(6.5 \%)$, (-)-gallocatechin-3-gallate $(4.0 \%)$, (-)-catechin-3-gallate $(0.2 \%)$, gallactocatechin $(0.2 \%)$, catechins $(1.1 \%)$ and is virtually caffeine free $(0.7 \%)$.

Transmission electron microscopy analysis. Immediately after excision, prostate samples of $\sim 2-3 \mathrm{~mm}$ of diameter were fixed in $1 \%$ osmium tetroxide solution buffered with sodium veronal buffer ( $\mathrm{pH}$ 7.4) for $1 \mathrm{~h}$. After being washed in Tyrode's solution, specimens were dehydrated in a graded series of acetone solutions and embedded in Durcupan (Fluka Chemie, Buchs, Switzerland).

Semithin sections ( $1 \mu \mathrm{m}$ thick) were stained with $0.5 \%$ toluidine blue, whereas ultrathin sections were stained with uranyl acetate and lead citrate (23) and observed by TEM (Philips 300, Philips, Eindhoven, The Netherlands). Histological and cytological controls excluded signs of distress and/or degenerative phenomena of the samples.

Cells, growth conditions and treatments. Murine tumourigenic prostate cancer TRAMP-C2 cells were purchased from the American Tissue Culture Collection and maintained in DMEM high glucose medium (Gibco, Grand Island, NY) containing 5\% FBS (Lonza Ltd., Basel, $\mathrm{CH}$ ), 5\% Nu-serum (Gibco), 1\% L-glutamine (Sigma Chemical Inc., St. Louis, MO), $0.005 \mathrm{mg} / \mathrm{ml}$ bovine Insulin (Sigma Chemical Inc.) and $10 \mathrm{nM}$ dihydrotestosterone (Sigma Chemical Inc.). Cells $\left(2,5 \times 10^{5}\right)$ were seeded in $\mathrm{T}_{75}$ flasks and cultured at $37^{\circ} \mathrm{C}$ in a $5 \% \mathrm{CO}_{2}$ atmosphere.

Twenty-four hours after seeding, cells were treated with $20 \mu \mathrm{g} / \mathrm{ml}$ of GTE. This represents a sub-lethal dose able to reduce drastically cell proliferation. Cell medium was replaced every $48 \mathrm{~h}$, and at each medium change GTE was added. Treated cells and their respective culture medium were collected after 7 days of treatment. Untreated cells, used as control, were harvested $48 \mathrm{~h}$ after seeding at a confluence comparable with that of treated cells.

Western blot analysis. Treated or untreated cells were washed twice with ice-cold PBS and scraped into ice-cold lysis buffer [50 mmol/1 Tris- $\mathrm{HCl}(\mathrm{pH} 8.0), 1 \%$ Triton X-100, $150 \mathrm{mmol}$ $\mathrm{NaCl}$ ] with protease inhibitors (Roche Diagnostic, Mannheim, 


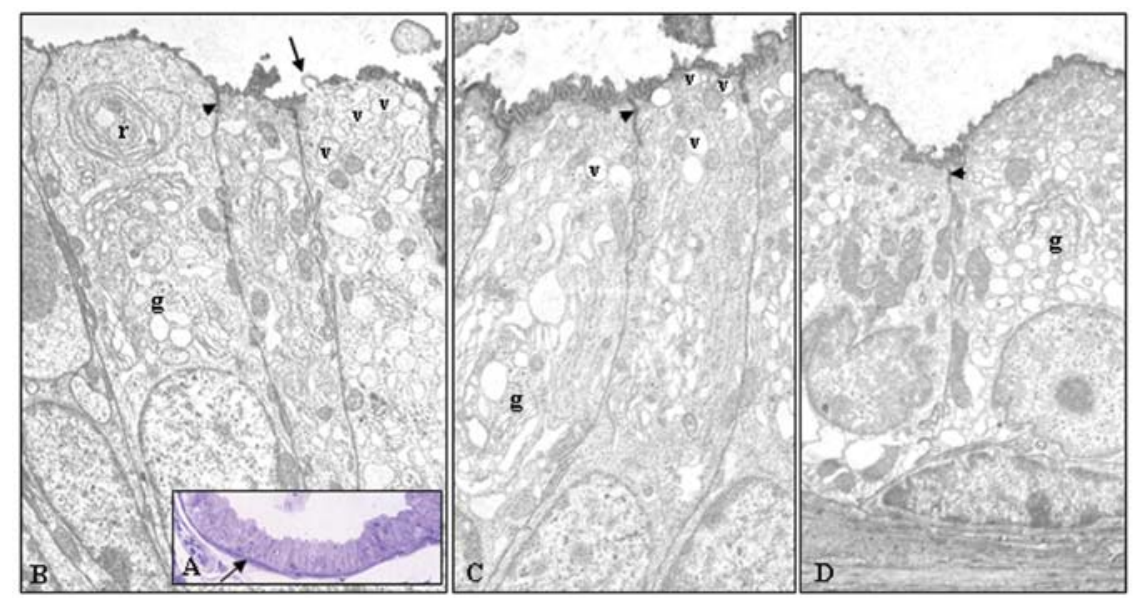

Figure 1. Twelve-week-old water-fed TRAMP mice: group I. The cuboidal-cylindrical epithelium (A, magnification x60, toluidine blue stain) protrudes often into small folds (PIN lesions) and rests on a thin basal lamina, externally lined by more smooth fibromuscular layers (arrow). TEM analysis (B, C and D, magnification x5670, x6750 and x4670, respectively) shows a marked synthetic activity denoted by well developed GA ( $\mathrm{g}$ ), concentric disposed ER tubules (r), numerous condensing vacuoles (v) and secretory granules with electrondense content. $\rightarrow$, exocytotic vacuoles; - , tight and gap junctions.

Germany) at the indicated times. Total protein lysates were resolved by SDS-PAGE, under reducing condition, $(50 \mu \mathrm{g}$ protein/lane), and electrophoretically transferred to PVDF membranes (Millipore Corporation, Billerica, MA). Transfer efficiency on the membranes was routinely monitored by $0.1 \%$ Red Ponceau S (Sigma Chemical). Membranes were probed with the following antibodies: anti-mouse $\beta$-clusterin (dilution 1:1000) from Santa Cruz Biotechnology (Santa Cruz, CA) and anti-human $\beta$-actin (dilution 1:2000) from Millipore Chemicon International (Temecula, CA).

\section{Results}

Group I: 12-week-old water-fed TRAMP mice. The epithelium of the dorso-lateral lobes of 12-week-old TRAMP mouse prostate exhibits PIN lesions. Many small and dense granules appear uniformly dispersed within a colloid-like secretion. Nuclei located in the medio-basal cytoplasm contain a central nucleolus and are surrounded by distinct dense secretion granules (Fig. 1A).

In the supranuclear cytoplasm many secretory vacuoles, electrondense secretory granules and a well-developed Golgi apparatus (GA), composed by curvilinear clusters of cisternae (g), are detected by TEM analysis (Fig. 1B, C and D). Many vacuoles located in the area between the GA and apical plasma membrane contain floccular material (v) with occasional dense core. Some vacuoles, characterized by a middle electron-dense secretion, and some secretory granules, frequently adhere and fuse to the luminal plasma membrane undergoing exocytosis (Fig. 1B, arrow). Dense bodies, similar to lysosomes, appear more numerous in the basal cytoplasm than in the Golgi area. It is worth noting that mostly tubular ER ( $\mathrm{r}$ ) is enlarged and, occasionally, displays a concentric arrangement (Fig. 1B). By histological and ultrastructural analyses the epithelial cells are characterized by a marked secretory activity.

Group II: 12-week-old TRAMP mice treated with GTE. The histological (Fig. 2A) and ultrastructural (Fig. 2B, C, D and E) features of the secretory prostatic epithelial cells of the 12-week-old TRAMP mice, after 4 weeks of GTE administration, are similar to those observed in the age-paired TRAMP mouse control group. In a typical picture of the secretory cells, a mature GA (g) exhibiting presecretory granules in the trans-face (Fig. 2B and C) is surmounted by many secretory vacuoles (v) with finely granular or amorphous material and small electron-dense granules. In the basal and perinuclear cytoplasm a tubular ER (Fig. 2C, r), lipofuscine-like dense bodies (arrowheads) and some phagosomes (ly) are also present (Fig. 2D and E).

Group III: 48-week-old water-fed TRAMP mice. The secretory epithelium of 48-week-old TRAMP mice exhibited nuclear stratification, micropapillar projections, scarce hyperchromatic and elongated nuclei (Fig. 3A). The GA (g, circles) in the epithelium lining the acini is not as well developed (Figs. 3B, D and E) as in the younger animals (group I); it is characterized by smaller expanded cisternae and vesicles. In the apical area of the cells, the cytoplasm, displaying a rather compact matrix, contains some secretory granules, electrondense vacuoles undergoing exocytosis (Fig. 3C, arrow) and secretory granules with electrondense content (Fig. 3D, arrowheads). Many dense bodies and secondary lysosomes (ly) can be seen in the basal cytoplasm (Fig. 3E). In comparison to the animals belonging to the group I we observed fewer interdigitations between the membranes of adjacent epithelial cells.

Group IV: 48-week-old TRAMP mice treated with GTE. Mice belonging to this experimental group exhibit only early signs of transformation. Most of the secretory acinar cells are characterized by a compressed cubical epithelium that occasionally forms modest folds, protruding in a very dilated acinar cavity, full of a high-dense granular material (Fig. 4A). Unlike the age-paired water-fed TRAMP mice (group III), many secretory cells present signs of apoptosis, but very few of them exhibit signs of mitosis.

As the most relevant ultrastructural finding, the cytoplasm, characterized by a structureless matrix of middle density, presents both the ER and GA strongly reduced in volume. In the 


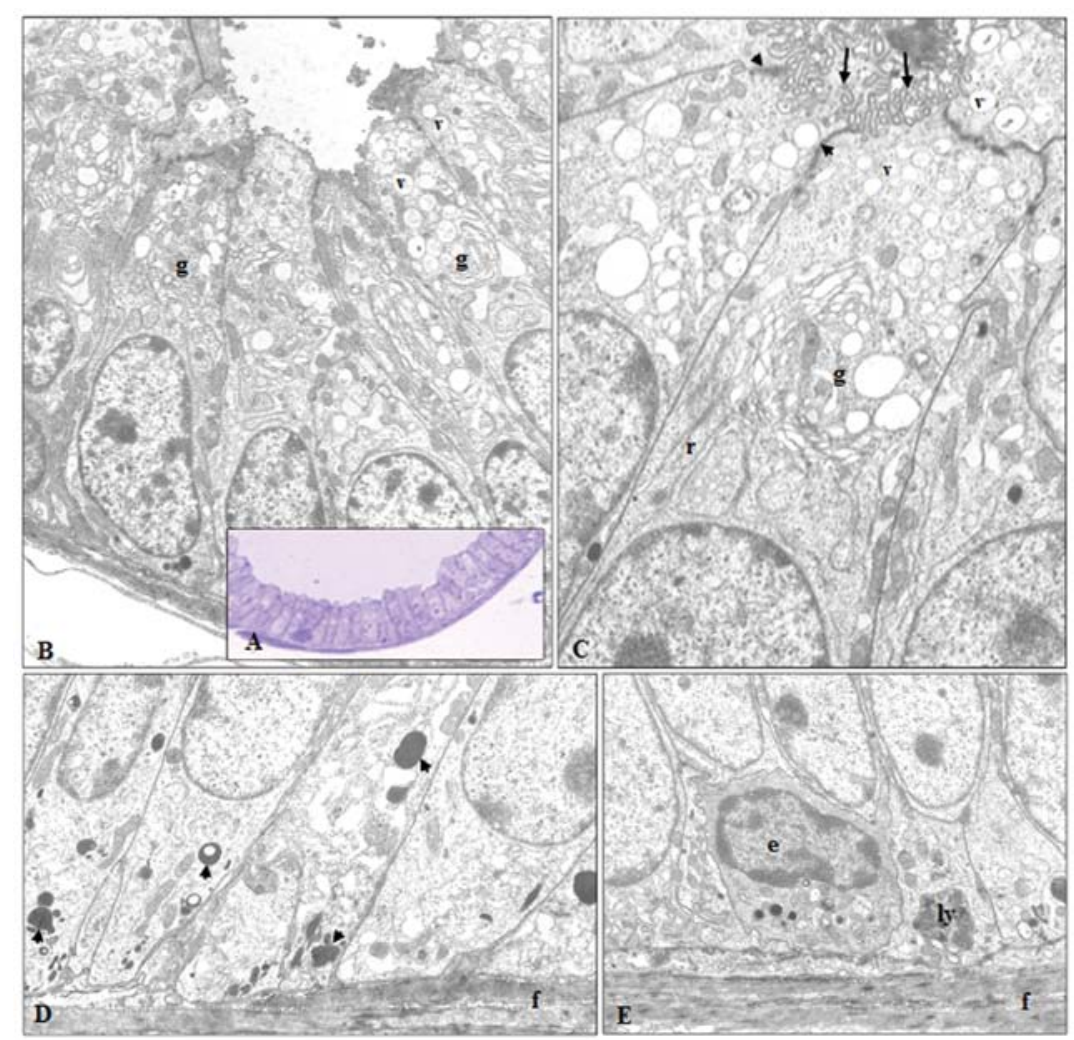

Figure 2. Twelve-week-old TRAMP mice treated with GTE: group II. Toluidine blue stain of the secretory cylindrical epithelium of the acinar adenomeres (A, magnification x72). TEM analysis (B and C, magnification x3500 and x6670, respectively) shows large GA (g), well developed rough ER (r) and numerous condensing vacuoles and secretory granules (v). Lipofuscine-like dense bodies (arrowheads) and some phagosomes (ly) were also distributed in the basal cytoplasm (D and E, magnification x6670 and x4670, respectively). Neuroendocrine cells, positive to the 5-hydroxytryptamine reaction (e), smooth muscle fibrocells (f). . , tight and gap junction.

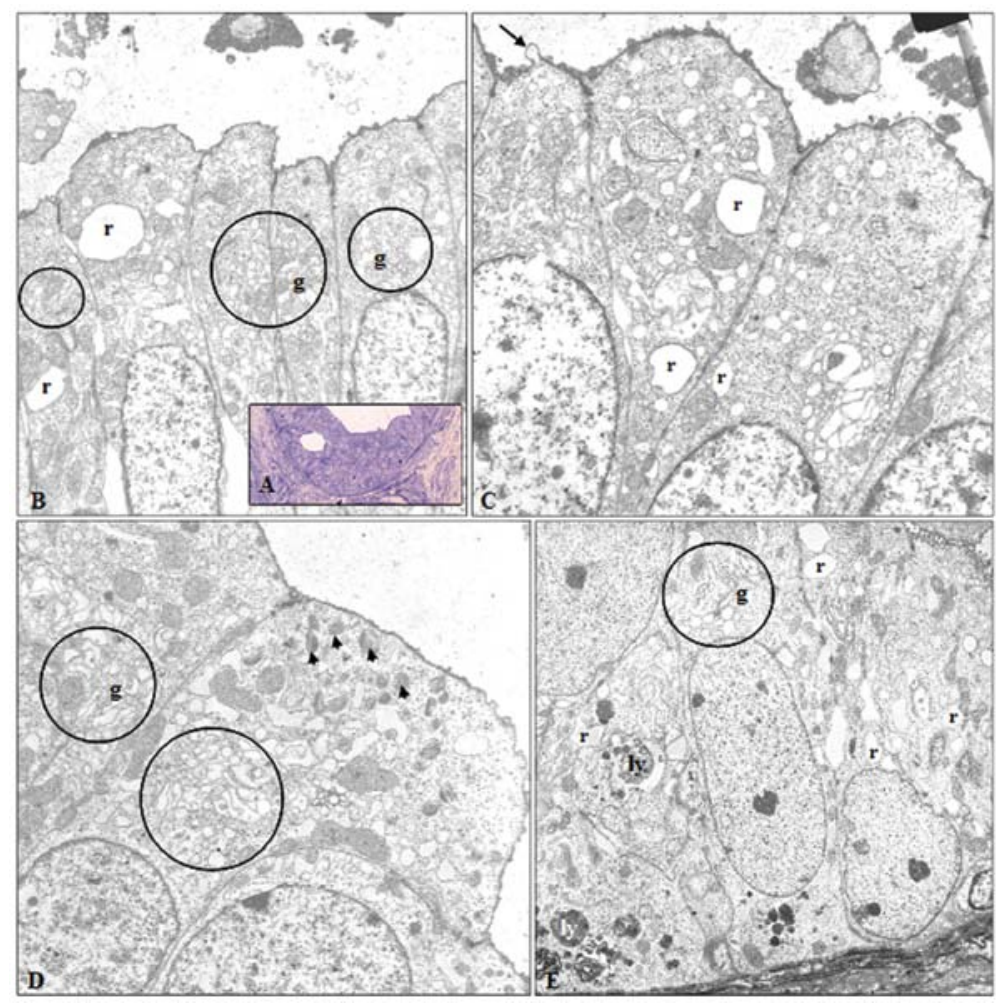

Figure 3. Forty-eight-week-old untreated TRAMP mice: group III. Toluidine blue stain of the secretory cylindrical epithelium (A) (magnification x200) show acinar adenomeres in a differentiated adenocarcinoma stage, few hyperchromatic nuclei and an increased number of apoptotic cells. TEM analysis (B, C and D, magnification x5000, x7000 and x7000, respectively) shows a large GA (g, circles), enlarged ER tubules (r) a low number of condensing vacuoles and granules, with dense content in the apical cytoplasm (arrowhead). Arrow, exocytotic vacuoles. Secondary lysosomes (ly) and dense bodies are detected in the basal cytoplasm. Endocrine cells (positive to the 5-hydroxytryptamine reaction) and basal cells are very scarce (E, magnification x7500). 

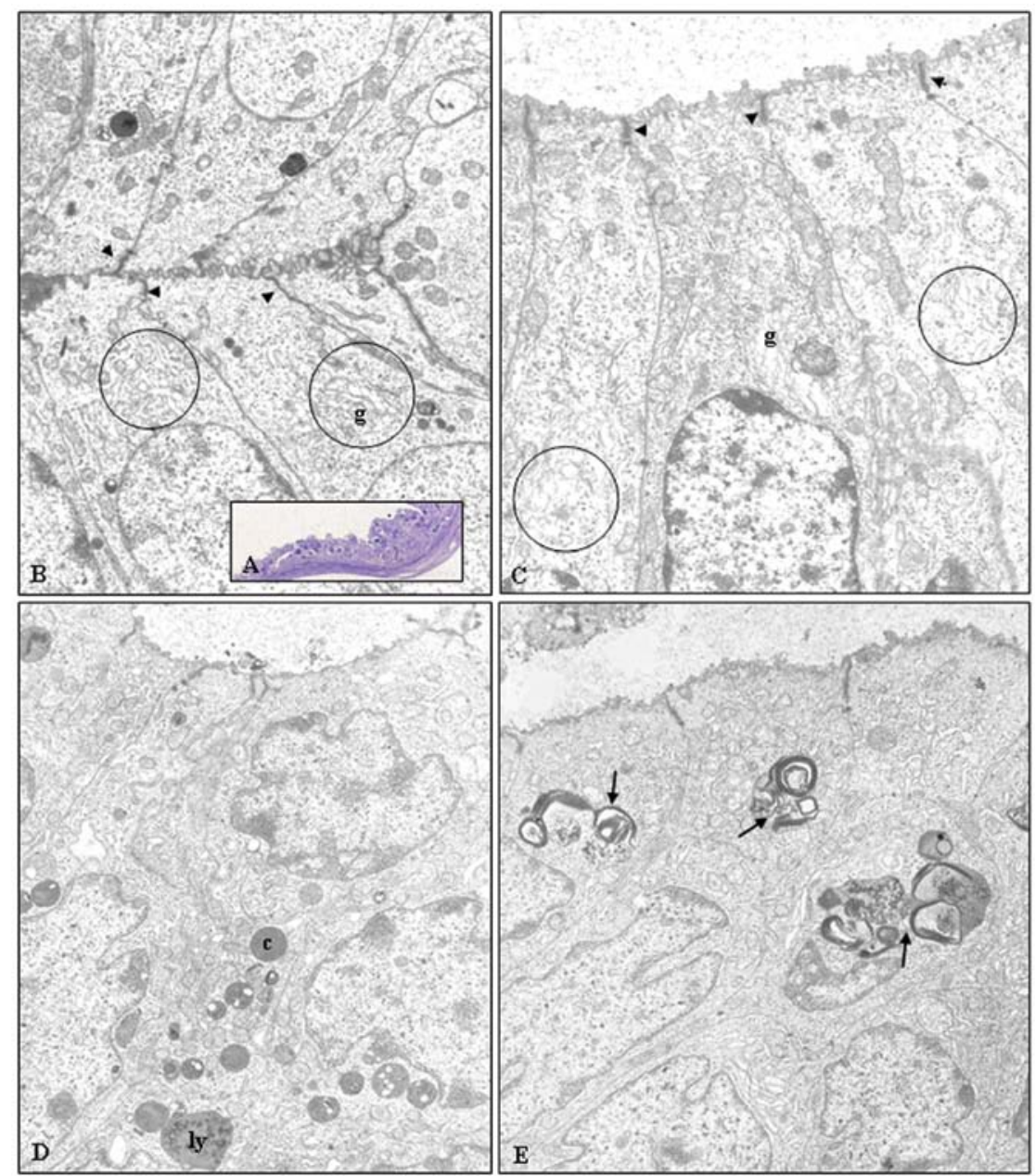

Figure 4. Forty-eight-week-old TRAMP mice treated with GTE: group IV. The secretory cuboidal-cylindrical epithelium (A, magnification x220) forms small folds and rests on a basal membrane lined by more smooth fibromuscular cells. TEM analysis (B and C, magnification x 9000 and x11250, respectively) shows a poorly developed GA (g, circles) and a tubular and poorly extended ER (r) and scarse condensing vacuoles and secretory vescicles. Numerous dense bodies (c), phagolysosomes (ly) large masses containing heterogeneous material and myelinic figures are also shown (arrows D and E, magnification x4560). $\boldsymbol{r},=$ desmosomes, tight and gap junctions.

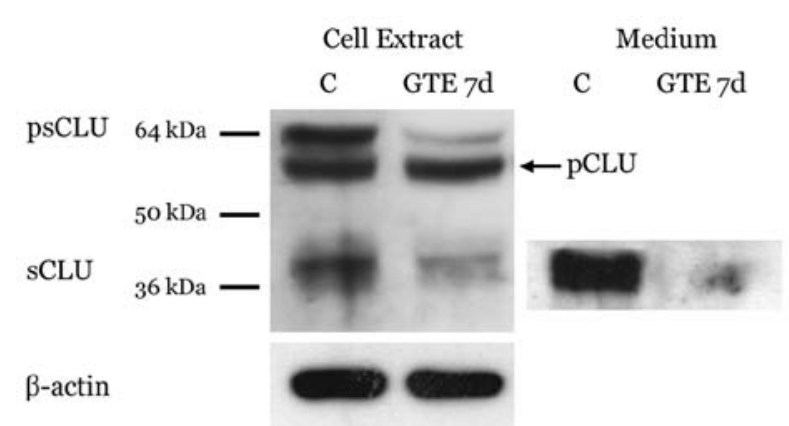

Figure 5. Clusterin biogenesis is modified in TRAMP-C2 cells treated with GTE. TRAMP-C2 cells were seeded and grown under standard conditions for $24 \mathrm{~h}$. Then cells were exposed to GTE $(20 \mu \mathrm{g} / \mu \mathrm{l})$ and harvested after 7 days of treatment. Cell culture medium were also collected. $\beta$-actin expression is provided to show equal loading of the samples. pCLU, precursor CLU $60 \mathrm{kDa}$; psCLU, presecretory CLU $64 \mathrm{kDa}$; sCLU, secreted CLU $40 \mathrm{kDa}$; C, control cells (untreated).

supranuclear area, the ER is constituted by very scant, flattened single tubules, lined by ribosomes. The GA (g, circles) consists in small clustered cisternae and in few secretory vesicles (Fig. 4B and $\mathrm{C}$ ). Likewise, in the apical cytoplasm, the condensing secretory vacuoles and the electron-dense secretory granules are scarce. Interestingly, we detected also many lysosomes (ly), fagolysosomes, fagosomes (Fig. 4D), big masses constituted of heterogeneous electrondense material, myelinic figures (Fig. 4E, arrows) and large cytoplasmic vacuoles, undergoing catabolic processes. The plasma membranes of the secretory epithelium also exhibit conspicuous lateral interdigitations with adjacent cells. Desmosomes and specialized junctional complexes are present in the apical cytoplasm (Fig. 4D and E).

The activity of ER-GA in TRAMP-C2 cells treated with GTE is impaired. To obtain functional proof that GTE administration causes a dysfunction of the ER-GA activity in the prostate epithelial cells, we monitored CLU expression in TRAMP-C2 cells, chronically treated with a sub-lethal concentration of GTE (20 $\mu \mathrm{g} / \mathrm{ml}$ for 7 days). Under physiological conditions, the CLU precursor (an uncleaved and unglycosylated peptide of $\sim 60 \mathrm{kDa}$ ) is modified (mainly by glycosylation) in the ER and 
proteolytically cleaved in the GA, to generate discrete $\alpha$ and $\beta$ chains (40 kDa each), prior to secretion.

As shown by WB analysis (Fig. 5), the pattern of expression of CLU in untreated TRAMP-C2 cells is characterized by three bands: the unmodified CLU precursor (pCLU, 60-kDa band), the CLU presecretory, uncleaved and partially glycosylated peptide (psCLU, 64-kDa band) and the mature form of CLU fully glycosylated (sCLU, 40-kDa band under reducing conditions). After 7 days of GTE treatment, both partially (psCLU, $64 \mathrm{kDa}$ ) and full processed (sCLU, $40 \mathrm{kDa}$ ) intracellular forms are strongly reduced, while the unmodified precursor (pCLU, $60 \mathrm{kDa}$ ) remains unmodified under the same experimental conditions.

We checked also for CLU content in the culture medium of control and treated cells. Consistenly with the results obtained with cellular protein lysates, we found that sCLU is drastically reduced in the medium of treated cells with respect to untreated ones.

\section{Discussion}

The basic structural definition of the TRAMP mouse prostate was previously reported by Kaplan-Lefko et al (2) and Gingrich et al (5) by means of optical microscopy, at different time intervals during PCa development. More recently, one of us showed the transendothelial passage of invasive tumour prostate cells from the extravasal matrix into the lymphatic vessel (24) in the same animal model.

In the present study, we extend the current knowledge of TRAMP mouse prostate morphology performing a detailed ultrastructural study of the secretory epithelial components of the prostatic acinar adenomeres in TRAMP mice, at 12 and 48 weeks of age. We paralleled the microscopic observation in age-matched TRAMP mice receiving GTE in tap water. Our present study design allowed us to gain insights into possible early ultrastructural alterations that might occur within the prostate gland in connection with the chemopreventive action of GTE.

We observed that the acinar adenomeres of 12-week-old TRAMP mice (group I) are characterized by scant preneoplastic and neoplastic lesions. Histological and ultrastructural analyses reveal a high secretory activity, accompanied by a colloid-like acinar secretion, which is uniformly enriched by small dense granules.

GTE administration to 12-week-old TRAMP mice (group II) during the last 4 weeks before sacrifice does not cause significant changes within the acinar cell components, as compared to age-matched control mice (group I). Forty-eight-week-old TRAMP mice (group III), exhibit a mild secretory activity, accompanied by a highly electrondense acinar cavity secretion, characterized by a strong affinity for haematoxylin. The different features of the prostatic secretion between 12- and 48-week-old mice may result from the reduced secretion activity, either due to animal aging or thickening of the secretory material caused by its slowed delivery through the tubular branching.

The epithelial acinar cells of TRAMP mice treated with GTE for 40 weeks before sacrifice (group IV), show significant modifications with respect to their age-matched untreated group (group III). Mice belonging to group IV exhibit only early patho- logical lesions with respect to time-matched untreated animals, possibly due to the chemopreventive action of GTE treatment.

We found a bold reduction of the organelles committed to the synthetic and secretory activities, ER and GA; consistently, the secretion condensing vacuoles and the secretory vesicles are very scarce. Many lysosomes and secondary lysosomes (fagolysosomes/auto-phagolysosomes) are also evident, together with large masses of heterogeneous electron-dense material and myelinic figures. The existence of large vacuoles containing cytoplasm undergoing degradation is also noteworthy. The overall picture strengthens the assumption that GTE administration can inhibit the condensing and secretory activities of the ER-GA.

Differently to that observed in group III, the plasma membranes of the secretory epithelium exhibit conspicuous lateral interdigitations with adjacent cells, desmosomes and specialized junctional complexes. It is well know that cell-cell junctional complexes play a crucial role in the establishment and maintenance of cell polarity. Reduced cell-cell interactions result in destruction of overall tissue architecture, a morphological hallmark of both early (loss of cell polarity and growth control) and late (invasion and metastasis) stages of tumour progression (25).

The morphological observation of a reduced activity of the ER-GA in the prostatic acinar cells is consistent with our in vitro findings of an altered CLU biogenesis in TRAMP-C2 cells, after treatment with GTE. The main product of the CLU gene is a mature secreted, glycosylated protein (sCLU), obtained through post-translational modification in the ER-GA of a CLU precursor protein (pCLU). In the present study we monitored pCLU and sCLU expression in TRAMP-C2 cells upon chronic administration of a sub-lethal dose of GTE to culture medium. The treatment quickly reduces sCLU expression, both in the cells and in the medium, while pCLU remains at the same level as in untreated cells. These experimental results are compatible with a marked slowdown of the biosynthetic and condensing processes of the ER-GA, that alters the processing velocity of pCLU and presents sCLU as a good marker for monitoring in vitro ER-GA functional status.

O'Sullivan et al reported that CLU processing through the ER-GA is drastically modified in MCF-7 cells, following treatment with Brefeldin A, a drug which induces stress of the ER via inhibition of ADP ribosylation factor (19). By using several complementary approaches Nizard et al, provided experimental evidence that partially processed CLU, under ER stressing conditions, can evade the secretion pathway being retrotraslocated from the ER-GA to the cytosol (20).

Our in vivo data show that GTE administration can drastically reduce the activity of the secretory pathway, possibly causing an imbalance of the protein trafficking. This phenomena would engulf the epithelial prostate cell cytoplasm with dense material. Recently, Wlodkowick et al have indicated that alterations of the ER-GA trafficking homeostasis is a signal for activation of digestion of exceeding endogenous material in the cell and also initiatiating and propagating cell death signaling (13).

It is rationale to speculate that the inhibition of the secretory activity in the TRAMP mice prostate after GTE administration might affect CLU processing, preventing its extensive glycosylation, cleavage and extracellular secretion, similarly to that in vitro. The consequent change in the pCLU/ 
sCLU ratio might take part in the processes leading GTE to counteract PCa progression (19,26-28). The above hypothesis is currently under investigation in our laboratory.

\section{Acknowledgments}

We thank Polyphenon Pharma, New York, USA for supplying Polyphenon E and Mr. Davide Dallatana, Section of Human Anatomy, University of Parma, for collaboration in drawing up the iconographic material. Funding information: FIL 20062008, University of Parma, Italy; AICR (UK) grant no. 06-711.

\section{References}

1. Greenberg NM, DeMayo F, Finegold MJ, et al: Prostate cancer in a transgenic mouse. Proc Natl Acad Sci USA 92: 3439-3443, 1995.

2. Kaplan-Lefko PJ, Chen TM, Ittmann MM, et al: Pathobiology of autochthonous prostate cancer in a pre-clinical transgenic mouse model. Prostate 55: 219-237, 2003.

3. Yeh IT, Reddick RL and Kumar AP: Malignancy arising in seminal vesicles in the transgenic adenocarcinoma of mouse prostate (TRAMP) model. Prostate 69: 755-760, 2009.

4. Tani Y, Suttie A, Flake GP, Nyska A and Maronpot RR: Epithelial-stromal tumor of the seminal vesicles in the transgenic adenocarcinoma mouse prostate model. Vet Pathol 42: 306-314, 2005.

5. Gingrich JR, Barrios RJ, Foster BA and Greenberg NM: Patho logic progression of autochthonous prostate cancer in the TRAMP model. Prostate Cancer Prostatic Dis 2: 70-75, 1999.

6. Gingrich JR, Barrios RJ, Kattan MW, Nahm HS, Finegold MJ and Greenberg NM: Androgen-independent prostate cancer progression in the TRAMP model. Cancer Res 57: 4687-4691, 1997.

7. Gingrich JR and Greenberg NM: A transgenic mouse prostate cancer model. Toxicol Pathol 24: 502-504, 1996.

8. Bettuzzi S, Brausi M, Rizzi F, Castagnetti G, Peracchia G and Corti A: Chemoprevention of human prostate cancer by oral administration of green tea catechins in volunteers with highgrade prostate intraepithelial neoplasia: a preliminary report from a one-year proof-of-principle study. Cancer Res 66: 1234-1240, 2006.

9. Gupta S and Mukhtar H: Green tea and prostate cancer. Urol Clin North Am 29: 49-57, 2002.

10. Caporali A, Davalli P, Astancolle S, et al: The chemopreventive action of catechins in the TRAMP mouse model of prostate carcinogenesis is accompanied by clusterin over-expression. Carcinogenesis 25: 2217-2224, 2004.

11. Bettuzzi S, Rizzi F and Belloni L: Clinical relevance of the inhibitory effect of green tea catechins $(\mathrm{GtCs})$ on prostate cancer progression in combination with molecular profiling of catechinresistant tumors: an integrated view. Pol J Vet Sci 10: 57-60, 2007.
12. Adhami VM, Siddiqui IA, Sarfaraz S, et al: Effective prostate cancer chemopreventive intervention with green tea polyphenols in the TRAMP model depends on the stage of the disease. Clin Cancer Res 15: 1947-1953, 2009.

13. Wlodkowic D, Skommer J, McGuinness D, Hillier C and Darzynkiewicz Z: ER-Golgi network - a future target for anticancer therapy. Leuk Res 33: 1440-1447, 2009.

14. Foster BA, Gingrich JR, Kwon ED, Madias C and Greenberg NM: Characterization of prostatic epithelial cell lines derived from transgenic adenocarcinoma of the mouse prostate (TRAMP) model. Cancer Res 57: 3325-3330, 1997.

15. Rizzi $\mathrm{F}$ and Bettuzzi S: The clusterin paradigm in prostate and breast carcinogenesis. Endocr Relat Cancer 17: R1-R17, 2010.

16. Shannan B, Seifert M, Leskov K, et al: Challenge and promise: roles for clusterin in pathogenesis, progression and therapy of cancer. Cell Death Differ 13: 12-19, 2006.

17. Burkey BF, deSilva HV and Harmony JA: Intracellular processing of apolipoprotein J precursor to the mature heterodimer. J Lipid Res 32: 1039-1048, 1991.

18. French LE, Chonn A, Ducrest D, et al: Murine clusterin: molecular cloning and mRNA localization of a gene associated with epithelial differentiation processes during embryogenesis. J Cell Biol 122: 1119-1130, 1993

19. O'Sullivan J, Whyte L, Drake J and Tenniswood M: Alterations in the post-translational modification and intracellular trafficking of clusterin in MCF-7 cells during apoptosis. Cell Death Differ 10: 914-927, 2003.

20. Nizard P, Tetley S, Le Drean Y, et al: Stress-induced retrotranslocation of clusterin/ApoJ into the cytosol. Traffic 8: 554-565, 2007.

21. Trougakos IP, Djeu JY, Gonos ES and Boothman DA: Advances and challenges in basic and translational research on clusterin. Cancer Res 69: 403-406, 2009.

22. Pucci S and Bettuzzi S: Chapter 3: The shifting balance between CLU forms during tumor progression. Adv Cancer Res 104: 25-32, 2009.

23. Reynolds ES: The use of lead citrate at high $\mathrm{pH}$ as an electronopaque stain in electron microscopy. J Cell Biol 17: 208-212, 1963.

24. Azzali G: Tumor cell transendothelial passage in the absorbing lymphatic vessel of transgenic adenocarcinoma mouse prostate. Am J Pathol 170: 334-346, 2007.

25. Mareel M and Leroy A: Clinical, cellular, and molecular aspects of cancer invasion. Physiol Rev 83: 337-376, 2003.

26. Moretti RM, Marelli MM, Mai S, et al: Clusterin isoforms differentially affect growth and motility of prostate cells: possible implications in prostate tumorigenesis. Cancer Res 67: 10325-10333, 2007.

27. Caccamo AE, Scaltriti M, Caporali A, et al: Nuclear translocation of a clusterin isoform is associated with induction of anoikis in SV40-immortalized human prostate epithelial cells. Ann NY Acad Sci 1010: 514-519, 2003.

28. Scaltriti M, Santamaria A, Paciucci R and Bettuzzi S: Intracellular clusterin induces G2-M phase arrest and cell death in PC-3 prostate cancer cells. Cancer Res 64: 6174-6182, 2004. 